\title{
In Vivo Cellular and Molecular Imaging
} Centers

National Cancer Institute

\section{Source}

National Cancer Institute. In Vivo Cellular and Molecular Imaging Centers. NCI Thesaurus.

Code C19961.

To foster multidisciplinary research on cellular and molecular imaging, $\mathrm{NCI}$ has established In Vivo Cellular and Molecular Imaging Centers. These centers will narrow the gap between the discovery of new cancer genes and intracellular pathways, and the translation of these discoveries into clinically useful, minimally invasive imaging approaches to expanding our understanding of cancer. 